\title{
members of commissions, boards, and committees
}

\section{EXECUTIVE COMMITTEE ${ }^{2}$}

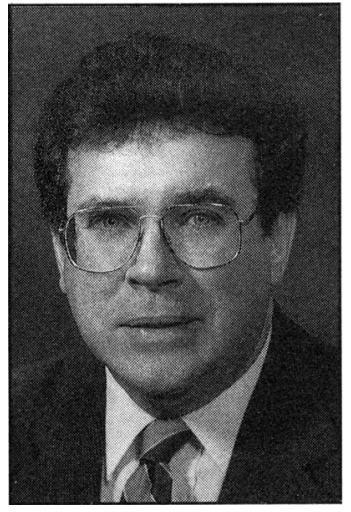

James R. Mahoney

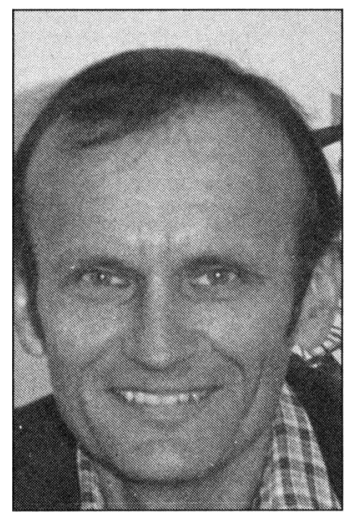

Robert J. Serafin

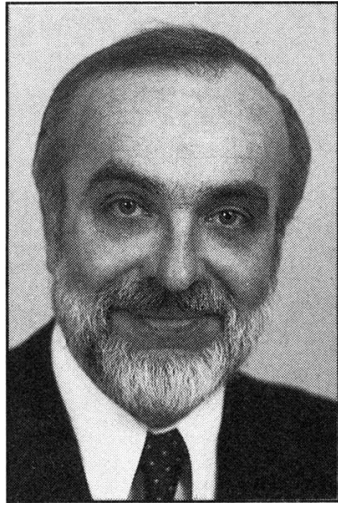

William D. Bonner

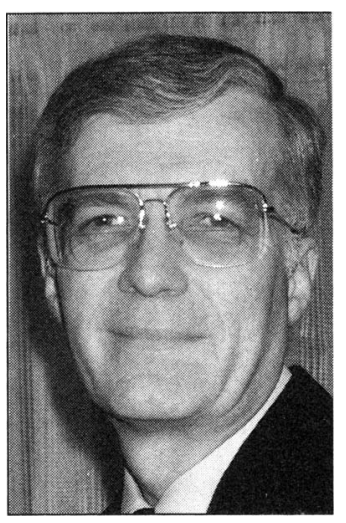

Paul D. Try

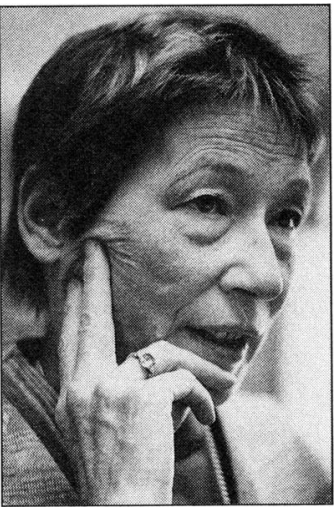

Joanne Simpson

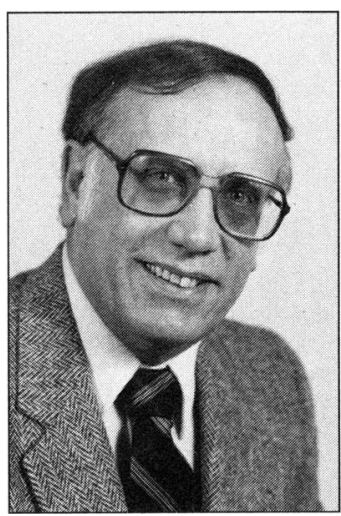

Richard E. Hallgren

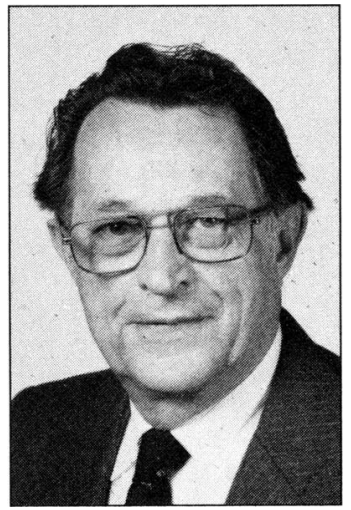

Roscoe R. Braham, Jr.

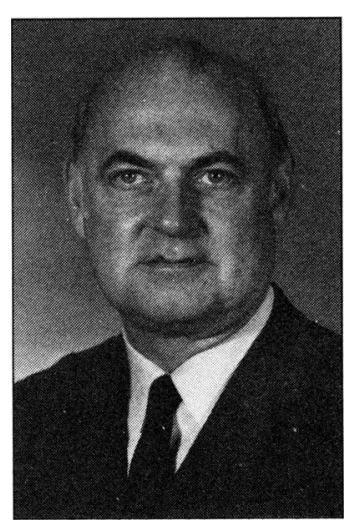

Kenneth C. Spengler

' The Executive Director is an ex officio member of all commissions, boards, and committees.

${ }^{2}$ Addresses listed on preceding page. 


\section{COMMITTEES OF THE EXECUTIVE COMMITTEE}

\section{Awards}

Nominating $^{3}$

\section{Admissions}

\section{History of the Atmospheric Sciences}

Chairman: Prof. Alfred K. Blackadar, Dept. of Meteorology, 503 Walker Building, Pennsylvania State University, University Park, PA 16802

Dr. Bruce A. Egan, ERT, 696 Virginia Road, Concord, MA 01742

Prof. Marvin A. Geller, Director, B106 Physics Bldg., Atmospheric Science, SUNY at Stony Brook, Stony Brook, NY 11974-3800

Dr. Syukuro Manabe, Geophysical-Fluid Dynamics Lab/NOAA, Princeton University, P.O. Box 308, Princeton, NJ 08542

Dr. Eugene M. Rasmusson, Dept. of Meteorology, University of Maryland, College Park, MD 20742-2425

Chairman: Dr. Margaret A. LeMone, National Center for Atmospheric Research, P.O. Box 3000, Boulder, CO 80307

Prof. John J. Cahir, Dept. of Meteorology, 503 Walker Building, Pennsylvania State University, University Park, PA 16802

Dr. Elbert W. Friday, Jr., Assistant Administrator for Weather Services, National Weather Service, NOAA, 8060 13th Street, Silver Spring, MD 20910

Prof. Richard H. Johnson, Dept. of Atmospheric Science, Colorado State University, Fort Collins, CO 80523

John W. Wilson, Malcolm Pirnie, Inc., 2 Corporate Park Drive, Box 751, White Plains, NY 10602

Chairman: Donald D. Grantham, Research Supervisory Meteorologist, Geophysics Laboratory (AFSC), GL/LYA, Hanscom AFB, MA 01886

James C. Barnes, Consultant, 6 Rose Court, Acton, MA 01720

Dr. Robert M. Cunningham, Rockwood Lane, Rte. 2, Box 166, Lincoln, MA 01773

Thomas P. McGuire, Travel Agents International, 200 West Cummings Park, Woburn, MA 01801

Prof. Keith L. Seitter, Dept. of Earth Science, University of Lowell, Lowell, MA 01854

Chairman: Dr. Claire L. Parkinson, Code 671, Goddard Space Flight Center, NASA, Greenbelt, MD 20771

Dr. George P. Cressman, Dept. of Meteorology, University of Maryland, College Park, MD 20742

Dr. Earl G. Droessler, Droessler Associates, P.O. Box 32247, Raleigh, NC 27622

Prof. Robert G. Fleagle, Dept. of Atmospheric Sciences, University of Washington, AK-40, Seattle, WA 98195

Prof. James R. Fleming, Program in Science \& Technological Studies, Dept. of Physics \& Astronomy, Colby College, Waterville, ME 04901

Prof. Julius London, Dept. of Astrophysical, Planetary and Atmospheric Sciences, P.O. Box 391, University of Colorado, Boulder, CO 80309-0391

Prof. Frederik L. Nebeker, Center for the History of Physics, American Institute of Physics, 335 East 45th St., New York, NY 10017

Dr. David L. Williamson, National Center for Atmospheric Research, P.O. Box 3000, Boulder, CO 80307-3000

\footnotetext{
${ }^{3}$ The AMS President serves ex officio on these committees.
} 
Investments $\mathbf{3}^{3,4}$

Chairman: Prof. Werner A. Baum, Dean, College of Arts and Sciences, Florida State University, Tallahassee, FL 32306

Col. Hazen H. Bedke, USAF/Ret., 2259 Laird Way, Salt Lake City, UT 84108

Eugene Bollay, 730 Woodland Rd., Santa Barbara, CA 93108

Dr. Thomas F. Malone, Saint Joseph College, West Hartford, CT 06117

\section{COMMITTEES OF THE COUNCIL}

Fellows ${ }^{3}$

\section{Sverdrup Gold Medal}

Public Policy
Chairman: Dr. Edward J. Zipser, Head, Dept. of Meteorology, Texas A\&M University, O \& M Building, Rm. 1204, College Station, TX 77843-3146

Brig. Gen. Albert J. Kaehn, Jr., (USAF/Ret.), Director, Eastern Region II, Government Systems Sector, Harris Corporation, 1201 East Abingdon Drive, Suite 300, Alexandria, VA 22314

Dr. Ronald D. McPherson, Director, National Meteorological Center, National Weather Service, NOAA, World Weather Building, Rm. 101, 5200 Auth Rd., Washington, DC 20233

Dr. Richard C. J. Somerville, Scripps Inst. of Oceanography, University of California-San Diego, Mail Code A-024, La Jolla, CA 92093

Prof. John M. Wallace, Dept. of Atmospheric Sciences, AK-40, University of Washington, Seattle, WA 98195

Chairman: Dr. Kirk Bryan, Jr., Geophysical Fluid Dynamics Lab, Environmental Research Lab, NOAA, Princeton University, P.O. Box 308, Forrestal Campus, Princeton, NJ 08540

Dr. Klaus Hasselmann, Max-Planck Institute, Bundestrasse 55, D-2000 Hamburg 13, FRG

Prof. Michael S. Longuet-Higgins, La Jolla Institute, 7855 Fay Ave., Suite 300, La Jolla, CA 92037

Prof. James J. O'Brien, Meteorology Annex, Florida State University, Tallahassee, FL 32306

Chairman: Dr. James R. Mahoney, Director, National Acid Precipitation Assessment Program, 722 Jackson Place, NW, Washington, DC 20503

Dr. William D. Bonner, Director, COMET, University Corporation for Atmospheric Research, P.O. Box 3000, Boulder, CO 80307-3000

Dr. Joanne Simpson, Chief Scientist for Meteorology, Code 612, Laboratory for Atmospheres, Goddard Space Flight Center, NASA, Greenbelt, MD 20771

Prof. Roscoe R. Braham, Jr., Dept. of Geophysical Sciences, The University of Chicago, 5734 South Ellis Ave., Chicago, IL 60637

Brig. Gen. Albert J. Kaehn, Jr., (USAF/Ret.), Director, Eastern Region II, Government Systems Sector, Harris Corporation, 1201 E. Abingdon Dr., Suite 300, Alexandria, VA 22314

Prof. Harold D. Orville, Head, Dept. of Meteorology, South Dakota School of Mines and Technology, 501 E. St. Joseph Street, Rapid City, SD 57701-3995

\footnotetext{
${ }^{3}$ The AMS President serves ex officio on these committees.

${ }^{4}$ The Chairman of the Planning Commission, the Executive Director, and the Secretary-Treasurer serve ex officio on this committee.
} 


\section{COMMISSION ON PROFESSIONAL AFFAIRS}

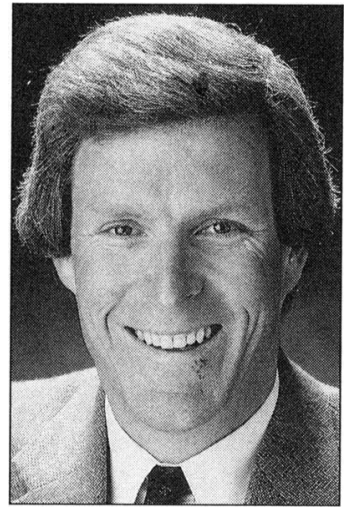

Robert T. Ryan
Chairman: Robert T. Ryan, WRC-TV, 4001 Nebraska Ave., NW, Washington, DC 20016 Members: Chairmen of the constituent Boards

\section{Board of Certified Consulting Meteorologists}

Board of Broadcast Meteorology
Chairman: Herbert D. Entrekin, Stone and Webster Inc., P.O. Box 5406, Denver, CO 80217 Richard R. Boyd, Radian Corporation, 10395 Old Placerville Rd., Sacramento, CA 958272506

John A. Ernst, NASA Headquarters, Code MOW, Washington, DC 20546

Dr. Timothy Spangler, COMET, University Corporation for Atmospheric Research, P.O. Box 3000, Boulder, CO 80307-3000

David B. Spiegler, 22 Fiske Rd., Lexington, MA 02173

Chairman: Paul H. Gross, WDIV-TV, 550 W. Lafayette Blvd., Detroit, MI 48212

Elliot Abrams, Accu-Weather Inc., 619 W. College Ave., State College, PA 16803

Paul Douglas, KARE-TV, 80811 Olson Memorial Highway, Minneapolis, MN 55427

Nicolas J. Gregory, WNYW-TV, 205 East 67th St., New York, NY 10021

Tom Loffman, KOVR-TV, 1014 Dunbarton Circle, Sacramento, CA 95825

Michael P. Nelson, KMOV-TV, 1 Memorial Dr., St. Louis, MO 63102

Harry V. Wappler, KIRO-TV, 2807 Third Ave., Seattle, WA 98121-7777

Chairman: Keith J. Brown CCM, North American Weather Consultants, 3761 South 700 East, Suite B, Salt Lake City, UT 84106

Todd Glickman, WSI Corporation, 4 Federal Street, Billerica, MA 01821

William H. Haggard, Climatological Consulting Corporation, Route 2, Box 372, Asheville, NC 28805

Thomas J. Lockhart, Meteorological Standards Inst., 841 Seventh Lane, P.O. Box 26, Fox Island, WA 98333 
Vincent Mirabella, Southern California Edison Co., P.O. Box 600, Rosemead, CA 91770

Jerry A. Williams, Oceanroutes Inc., 680 W. Maude Ave., Sunnyvale, CA 94086-3518

\section{Board for Operational Government Meteorologists}

Chairman: Larry B. Dunn, National Weather Service Western Region, Scientific Services Division, 125 South State Street, P.O. Box 11188, Salt Lake City, UT 84147

Cecilia A. Askue, Major, U.S. Air Force, Headquarters AWS/XTRS, Scott AFB, IL 62225-5008 Benjamin C. Hablutzel, National Weather Service Forecast Office, P.O. Box 29879, Honolulu, HI 96820

Chet Henricksen, National Weather Service Forecast Office, Rm. 9258, 600 Arch St., Philadelphia, PA 19106

Donald A. Mautner, Commander, U.S. Navy, FLENUMOCEANCEN Code 03, Monterey, CA 93943-5005

Ken Mielke, Chief, National Weather Service Western Region, Scientific Services Division, 125 South State Street, P.O. Box 11188, Salt Lake City, UT 84147

\section{PUBLICATIONS COMMISSION}

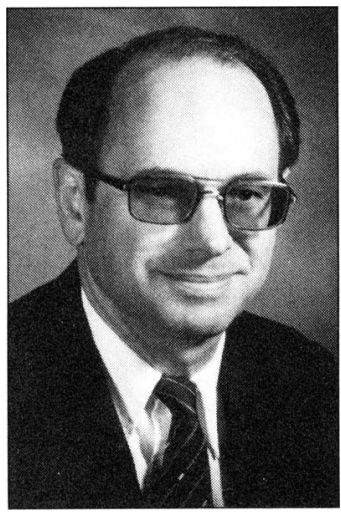

Chairman: Prof. Richard E. Orville, Dept. of Atmospheric Sciences, State University of New York-Albany, 1400 Washington Ave., Albany, NY 12222

Members: Editors-in-Chief of periodicals and serials

Members at Large:

Prof. Alfred K. Blackadar, Dept. of Meteorology, 503 Walker Bldg., Pennsylvania State University, University Park, PA 16802

Dr. Ronald D. McPherson, Director, National Meteorological Center, National Weather Service, NOAA, World Weather Building, Rm. 101, 5200 Auth Rd., Washington, DC 20233

Dr. Norman A. Phillips, 18 Edward Lane, Merrimack, NH 03054

Richard E. Orville 


\section{Board of the Journal of the Atmospheric Sciences}

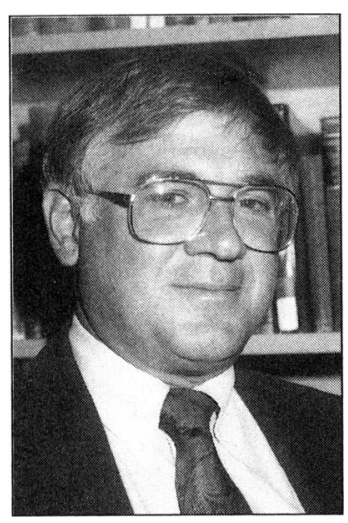

Robert L. Gall
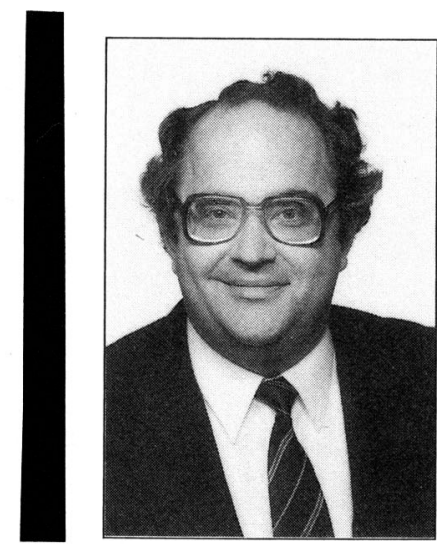

Philip Krider
Co-Chief Editor: Prof. Robert L. Gall, Institute of Atmospheric Physics, PAS Building \#81, Room 542, University of Arizona, Tucson, AZ 85721

Co-Chief Editor: Prof. Philip Krider, Chairman, Dept. of Meteorology and Director of the Institute of Atmospheric Physics, PAS Building \#81, University of Arizona, Tucson, AZ 85721

\section{JAS Editors:}

Dr. G. Brant Foote, National Center for Atmospheric Research, P.O. Box 3000, Boulder, CO 80307

Dr. Isaac M. Held, NOAA/Geophysical Fluid Dynamics Laboratory, Princeton University, P.O. Box 308, Princeton, NJ 08542

Prof. R. T. Pierrehumbert, Dept. of Geophysical Sciences, The University of Chicago, 5734 South Ellis Ave., Chicago, IL 60637

Alan R. Plumb, Professor of Meteorology, Massachusetts Institute of Technology, Cambridge, MA 02139

Prof. Graeme L. Stephens, Dept. of Atmospheric Sciences, Colorado State University, Fort Collins, CO 80525

JAS Associate Editors:

Richard A. Anthes, National Center for Atmospheric Research, Boulder, CO Akio Arakawa, University of California at Los Angeles, Los Angeles, CA Barney J. Conrath, Goddard Space Flight Center, NASA, Greenbelt, MD Kerry A. Emanuel, Massachusetts Institute of Technology, Cambridge, MA J. Michael Fritsch, The Pennsylvania State University, University Park, PA Marvin A. Geller, Goddard Space Flight Center, NASA, Greenbelt, MD Dennis L. Hartmann, University of Washington, Seattle, WA

Andrew J. Heymsfield, National Center for Atmospheric Research, Boulder, CO James R. Holton, University of Washington, Seattle, WA Brian J. Hoskins, University of Reading, Reading, United Kingdom Robert A. Houze, Jr., University of Washington, Seattle, WA Eugenia Kalnay, NOAA/National Meteorological Center, Washington, DC Steven E. Koch, Goddard Space Flight Center, NASA, Greenbelt, MD Colleen A. Leary, Texas Tech University, Lubbock, TX Margaret A. LeMone, National Center for Atmospheric Research, Boulder, CO Mitchell Moncrieff, National Center for Atmospheric Research, Boulder, CO David A. Randall, Colorado State University, Fort Collins, CO Edward S. Sarachik, University of Washington, Seattle, WA Ronald B. Smith, Yale University, New Haven, CT Susan Solomon, National Oceanic and Atmospheric Administration, Boulder, CO Duane E. Stevens, Colorado State University, Fort Collins, CO Peter J. Webster, The Pennsylvania State University, State College, PA Michio Yanai, University of California at Los Angeles, Los Angeles, CA 


\section{Board of the Journal of Applied Meteorology}

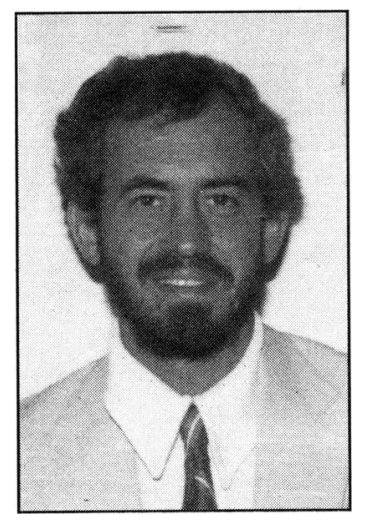

Steven R. Hanna

Chief Editor: Dr. Steven R. Hanna, Sigma Research Corporation, 533 Hill Rd., Boxborough, MA 01719

JAM Editors:

Dr. Robert F. Adler, Code 612, Goddard Space Flight Center, NASA, Greenbelt, MD 20771

Dr. Gabor Vali, Dept. of Atmospheric Sciences, University of Wyoming, P.O. Box 3038, University Station, Laramie, WY 82071

\section{JAM Associate Editors:}

Robert D. Bornstein, San Jose State University, San Jose, CA

Richard E. Carbone, National Center for Atmospheric Research, Boulder, CO

Richard J. Doviak, NOAA/National Severe Storms Laboratory, Norman, OK

Henry E. Fleming, NOAA/National Environmental Satellite Data, and Information Service,

Washington, DC

Kenneth S. Gage, NOAA/Environmental Research Laboratories, Boulder, CO

Charles A. Knight, National Center for Atmospheric Research, Boulder, CO

Brian K. Lamb, Washington State University, Pullman, WA

Paul W. Mielke, Jr., Colorado State University, Fort Collins, CO

Thomas R. Parish, University of Wyoming, Laramie, WY

Robert S. Schemenauer, Atmospheric Environment Service, Downsview, Ontario, Canada Roland B. Stull, University of Wisconsin, Madison, WI

Joel Susskind, Goddard Space Flight Center, NASA, Greenbelt, MD

Akula Venkatram, ENSR, Inc., Camarillo, CA

Jeffrey C. Weil, National Center for Atmospheric Research, Boulder, CO

\section{Board of the Journal of Physical Oceanography}

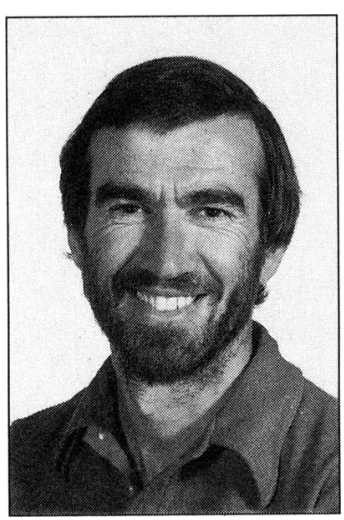

Peter R. Gent

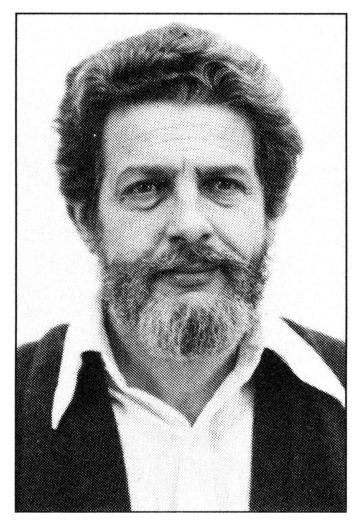

Eli Joel Katz
Co-Editor: Dr. Peter R. Gent, National Center for Atmospheric Research, P.O. Box 3000, Boulder, CO 80307

Co-Editor: Dr. Eli Joel Katz, Lamont-Doherty Geological Observatory of Columbia University, Palisades, NY 10964 
JPO Associate Editors:

Kenneth H. Brink, Woods Hole Oceanographic Institution, Woods Hole, MA

Claude Frankignoul, Universite Pierre et Marie Curie, Paris, France

Ann E. Gargett, Institute of Ocean Science, British Columbia, Canada

Robert L. Haney, Naval Postgraduate School, Monterey, CA

Norden E. Huang, Goddard Space Flight Center, NASA, Greenbelt, MD

Peter D. Killworth, University of Oxford, Oxford, United Kingdom

Robert A. Knox, Scripps Institution of Oceanography, La Jolla, CA

William G. Large, University of British Columbia, Vancouver, British Columbia, Canada

Paola Malanotte-Rizzoli, Massachusetts Institute of Technology, Cambridge, MA

Peter Muller, University of Hawaii, Honolulu, HI

Lawrence A. Mysak, McGill University, Montreal, Quebec, Canada

Hsien Wang Ou, Lamont-Doherty Geological Observatory of Columbia University, Palisades, NY

Edward S. Sarachik, University of Washington, Seattle, WA

\section{Board of the Monthly Weather Review}

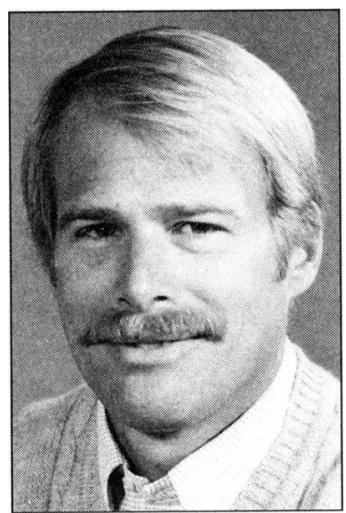

Joseph B. Klemp

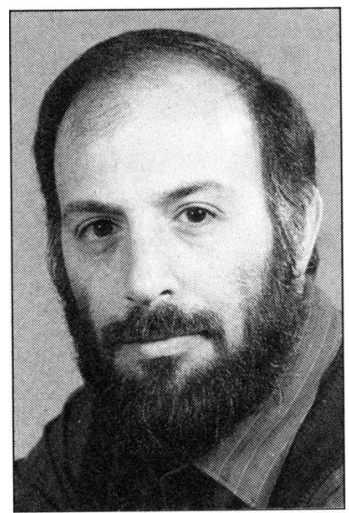

Richard Rotunno
Co-Chief Editor: Dr. Joseph B. Klemp, National Center for Atmospheric Research, Box 3000, Boulder, CO 80307

Co-Chief Editor: Dr. Richard Rotunno, National Center for Atmospheric Research, Box 3000, Boulder, CO 80307

MWR Editors:

Dr. Bruce A. Albrecht, Dept. of Meteorology, Walker Building, The Pennsylvania State University, University Park, PA 16802

Prof. Frederick Sanders, 9 Flint St., Marblehead, MA 01945

MWR Associate Editors:

Gary M. Barnes, National Center for Atmospheric Research, Boulder, CO Frederick H. Carr, University of Oklahoma, Norman, OK Roger W. Daley, Canadian Climate Centre, Downsview, Ontario, Canada Randall M. Dole, Massachusetts Institute of Technology, Cambridge, MA Kerry A. Emanuel, Massachusetts Institute of Technology, Cambridge, MA Steven K. Esbensen, Oregon State University, Corvallis, OR John R. Gyakum, McGill University, Montreal, Quebec, Canada

Daniel Keyser, State University of New York at Albany, Albany, NY

John M. Lewis, NOAA/ERL/National Severe Storms Laboratory, Norman, OK

Robert A. Maddox, NOAA/ERL/National Severe Storms Laboratory, Norman, OK

Larry Mahrt, Oregon State University, Corvallis, OR

Clifford F. Mass, University of Washington, Seattle, WA

Dr. Ronald D. McPherson, Director, National Meteorological Center, National Weather Service, NOAA, World Weather Building, Rm. 101, 5200 Auth Rd., Washington, DC

Robert G. Miller, NOAA/National Weather Service, Techniques Development Laboratory, Silver Spring, MD

Peter S. Ray, Florida State University, Tallahassee, FL

Lloyd J. Shapiro, NOAA/ERL/Atlantic Oceanographic and Meteorological Laboratories, Miami, FL

Andrew N. Staniforth, Atmospheric Environment Service, Dorval, Quebec, Canada

Joseph J. Tribbia, National Center for Atmospheric Research, Boulder, CO 


\section{Board of the Journal of Atmospheric and Oceanic Technology}

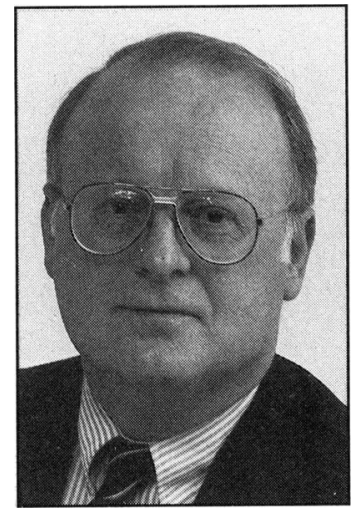

Thomas B. Sanford

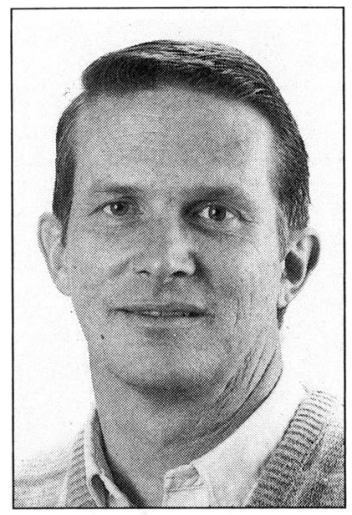

William A. Cooper
Co-Chief Editor/Oceanic Technology: Prof. Thomas B. Sanford, Applied Physics Laboratory, University of Washington, 1013 Northeast 40th St., Seattle, WA 98105 Co-Chief Editor/Atmospheric Technology: Dr. William A. Cooper, National Center for Atmospheric Research, P.O. Box 3000, Boulder, CO 80307

\section{JAOT Associate Editors:}

Donald T. Acheson, NOAA/National Weather Service, Silver Spring, MD

Otis B. Brown, Rosenstiel School of Marine and Atmospheric Sciences, University of Miami, Miami, FL

Douglas R. Caldwell, School of Oceanography, Oregon State University, Corvallis, OR

Hugh J. Christian, Marshall Space Flight Center, NASA, Huntsville, AL

Robert W. Corell, National Science Foundation, Washington, DC

Russ E. Davis, Scripps Institution of Oceanography, University of California at San Diego, San Diego, CA

Carl A. Friehe, University of California, Irvine, CA

William G. Large, National Center for Atmospheric Research, Boulder, CO

Donald H. Lenschow, National Center for Atmospheric Research, Boulder, CO Lawrence F. Radke, University of Washington, Seattle, WA

Brian A. Ridley, National Center for Atmospheric Research, Boulder, CO

Robert S. Schemenauer, Atmospheric Environment Service, Downsview, Ontario, Canada

Robert C. Spindel, Applied Physics Laboratory, University of Washington, Seattle, WA

Richard G. Strauch, NOAA/Environmental Research Laboratories, Boulder, CO

Dennis W. Thomson, The Pennsylvania State University, University Park, PA

Raymond F. Weiss, Scripps Institution of Oceanography, University of California at San Diego, San Diego, CA

Frank Wentz, Remote Sensing Systems, Inc., Sausalito, CA

Thomas T. Wilheit, Texas A\&M University, College Station, TX

Dusan S. Zrnic, NOAA/National Severe Storms Laboratory, Norman, OK 


\section{Board of Weather and Forecasting}

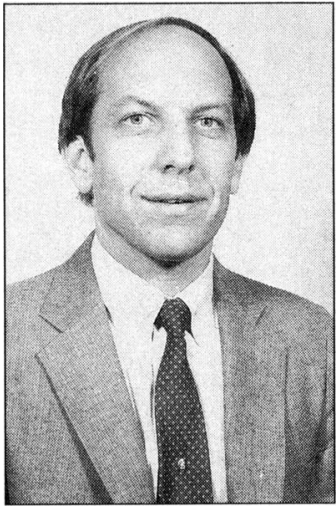

Louis W. Uccellini

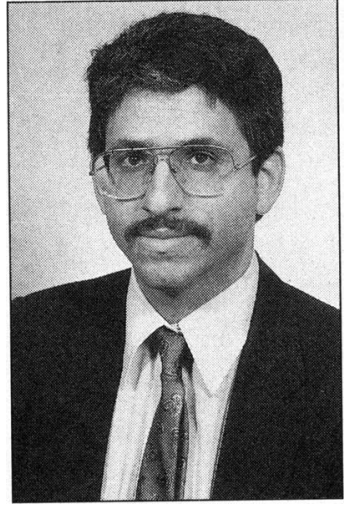

Paul J. Kocin
Co-Chief Editor: Dr. Louis W. Uccellini, W/NMC3, World Weather Building, Rm. 410, 5200 Auth Rd., Camp Springs, MD 20746

Co-Chief Editor: Mr. Paul J. Kocin, W/NMC3, World Weather Building, Rm. 410, 5200 Auth Rd., Camp Springs, MD 20746

WAF Associate Editors:

Stanley L. Barnes, NOAA/Environmental Research Laboratories, Boulder, CO Lance Bosart, State University of New York at Albany, Albany, NY

Frederick H. Carr, University of Oklahoma, Norman, OK

Gary M. Carter, NOAA/National Weather Service, Bohemia, NY

Charles A. Doswell, III, NOAA/ERL/National Severe Storms Laboratory, Norman, OK

Gary K. Grice, NOAA/National Weather Service, Fort Worth, TX

Robert H. Johns, National Severe Storms Forecast Center, Kansas City, MO

Laurence G. Lee, NOAA/National Weather Service, Raleigh-Durham Airport, Morrisville, NC

Dennis H. McCarthy, NOAA/National Weather Service, Saint Charles, MO Allan H. Murphy, Oregon State University, Corvallis, OR

Patricia M. Pauley, University of Wisconsin-Madison, WI

Ralph A. Petersen, NOAA/National Meteorological Center, Washington, DC Russell Schneider, University of Wisconsin-Madison, WI

Edward J. Szoke, National Center for Atmospheric Research, Boulder, CO

Carlyle H. Wash, Naval Postgraduate School, Monterey, CA

\section{Board of the Journal of Climate}

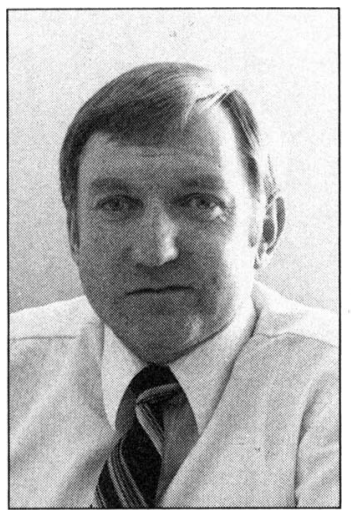

Chief Editor: Dr. Peter J. Lamb, Climate and Meteorology Section, Illinois State Water Survey, 2204 Griffith Dr., Champaign, IL 61821

Editor: Dr. Robert E. Livezey, W/NMC 51, Climate Analysis Center, NWS/NOAA, Rm. 604, 5200 Auth Rd., Washington, DC 20233

Editor: Dr. Peter H. Stone, Center for Meteorology and Physical Oceanography, Massachusetts Institute of Technology, Rm. 54-1718, Cambridge, MA 02139

Peter J. Lamb 


\section{JC Associate Editors:}

Eric J. Barron, The Pennsylvania State University, University Park, PA

Mark A. Cane, Lamont-Doherty Geological Observatory of Columbia University, Palisades, NY

James A. Coakley, Jr., Oregon State University, Corvallis, OR

Stewart J. Cohen, Atmospheric Environment Service, Downsview, Ontario, Canada

Robert E. Dickinson, National Center for Atmospheric Research, Boulder, CO

Edward S. Epstein, National Weather Service, NOAA, Climate Analysis Center, Washington, DC

David S. Gutzler, Atmospheric and Environmental Research, Inc., Cambridge, MA

Thomas R. Karl, National Climatic Data Center, Asheville, NC

Michael C. MacCracken, Lawrence Livermore Laboratory, University of

California, Livermore, CA

Michael B. Richman, Illinois State Water Survey, Champaign, IL

Claes G. H. Rooth, Rosensteil School, University of Miami, Miami, FL

Chester F. Ropelewski, National Weather Service, NOAA, Climate Analysis Center, Washington, DC

Richard D. Rosen, Atmospheric and Environmental Research, Inc., Cambridge, MA

Norman Rosenberg, Resources for the Future, Washington, DC

V. Kerry Smith, North Carolina State University, Raleigh, NC

Charles W. Stockton, University of Arizona, Tucson, AZ

Larry L. Stowe, NOAA/National Environmental Satellite, Data, and Information Service, Washington, DC

Kevin E. Trenberth, National Center for Atmospheric Research, Boulder, CO

Compton J. Tucker, Goddard Space Flight Center, NASA, Greenbelt, MD

John E. Walsh, University of Illinois, Urbana, IL

Thompson Webb, III, Brown University, Providence, RI

\section{Board of Meteorological Monographs}

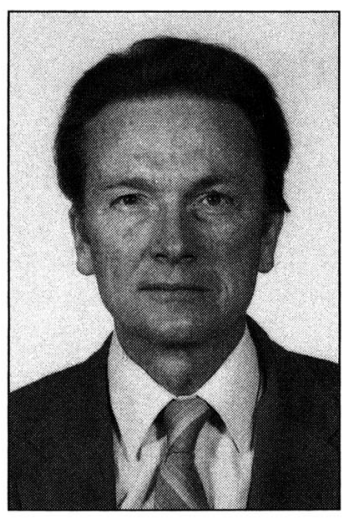

Editor: Prof. Donald R. Johnson, Space, Science \& Engineering Center, University of Wisconsin-Madison, 1225 West Dayton Street, Madison, WI 53706

Monograph Associate Editors:

Robert C. Beardsley, Woods Hole Oceanographic Institution, Woods Hole, MA James R. Holton, University of Washington, Seattle, WA

Richard E. Orville, State University of New York at Albany, Albany, NY

Jesse J. Stephens, Florida State University, Tallahassee, FL

Donald R. Johnson 


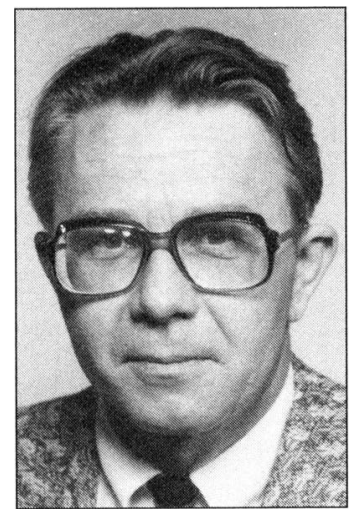

Historical Monograph Series Editor:

Dr. Ronald C. Taylor, Physical Meteorology Program, Division of Atmospheric Sciences, National Science Foundation, Washington, DC 20550

Ronald C. Taylor

\section{Bulletin of the American Meteorological Society}

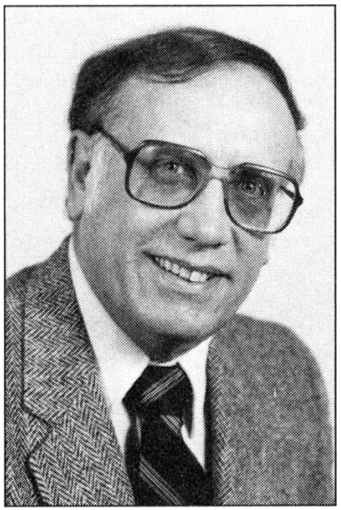

Editor: Dr. Richard E. Hallgren, Executive Director, American Meteorological Society, 45 Beacon Street, Boston, MA 02108

Richard D. Hallgren

\section{Board of Meteorological and Geoastrophysical Abstracts}

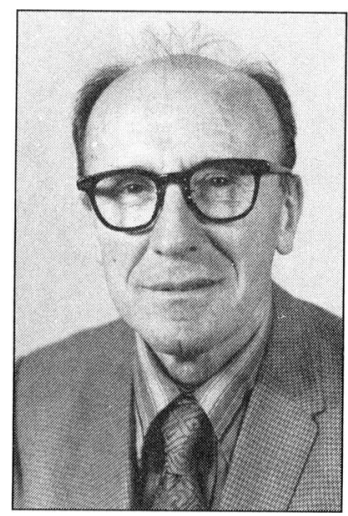

IN MEMORIAM

Malcolm Rigby

Editor-1950-89 


\section{SCIENTIFIC AND TECHNOLOGICAL ACTIVITIES COMMISSION ${ }^{7}$}

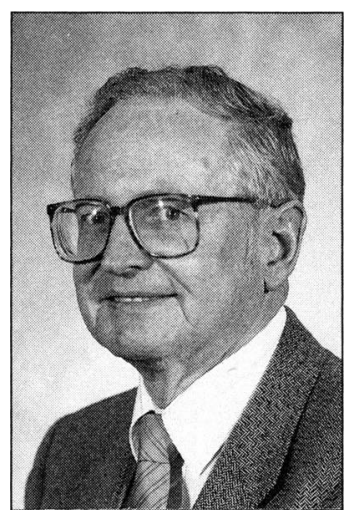

Harold D. Orville

Chairman: Prof. Harold D. Orville, Head, Institute of Atmospheric Science, South Dakota School of Mines and Technology, 501 E. St. Joseph Street, Rapid City, SD 57701

Members: Chairmen of the Scientific and Technological Activities Committees

\section{Agricultural and Forest Meteorology}

Chairman: Dr. Katharine B. Perry, Dept. of Horticulture, North Carolina State University, P.O. Box 7609, Raleigh, NC 27695-7609

Dr. William Bland, Texas A\&M University, Blackland Research Center, 808 East Blackland Rd., Temple, TX 76502

Prof. Paul W. Brown, Dept. of Soil and Water Science, 429 Shantz Bldg., No. 38, University of Arizona, Tuscon, AZ 85721

Dr. Bhaskar Choudhury, Code 620, Laboratory for Terrestrial Physics, Goddard Space Flight Center, NASA, Greenbelt, MD 20771

Prof. Richard H. Grant, Dept. of Agronomy, Life Sciences Building, Purdue University, West Lafayette, IN 47907-7899

Prof. Lawrence E. Hipps, Dept. of Soil Science and Biometeorology, Utah State University, Logan, UT 84322-4840

Dr. Steven E. Hollinger, Climate \& Meteorology Section, Illinois State Water Survey, 2204 Griffith Dr., Champaign, IL 61820-7495

Dr. David R. Miller, University of Connecticut, Dept. of Renewable Natural Resources, WBY Bldg., Rm. 308 U-87 1376 Storrs Rd., Storrs, CT 06268

Bertrand D. Tanner, Campbell Scientific, P.O. Box 551, Logan, UT 84321

\section{Applied Climatology}

Chairman: Thomas R. Karl, Chief, Climate Perspectives Branch, National Climatic Data Center, NOAA, Federal Bldg., Asheville, NC 28801

Dr. William E. Easterling, Resources for the Future, 1616 P Street, NW, Washington, DC 20036 Col. William B. Freeman, Jr., U.S. Air Force Environmental Technical Applications Center, Scott AFB, IL 62225-5438

Dr. Thomas R. Heddinghaus, NOAA/USDA, Joint Agricultural Weather Facility, USDA South Agricultural Bldg., Rm. 5884, Washington, DC 20250

Dr. Laurence S. Kalkstein, Center for Climatic Research, Dept. of Geography, University of Delaware, Newark, DE 19716

Prof. Robert A. Muller, Dept. of Geography and Anthropology, Louisiana State. University, Baton Rouge, LA 70803

Dr. Kelly T. Redmond, Office of the State Climatologist, Climate Research Institute, Oregon State University, Corvallis, OR 97331

Herman H. Shugart, Dept. of Environmental Sciences, University of Virginia, Charlottesville, VA 22903

'The chairman of STAC and the executive director of AMS are ex officio members of all STAC committees. 
Dr. Roger Street, Conservation \& Protection, Environment Canada, 25 St. Clair Ave. East, Toronto, Ontario, Canada M4T 1M2

Dr. Donald A. Wilhite, Dept. of Agricultural Meteorology, 241 L.W. Chase Hall, University of Nebraska, Lincoln, NE 68583-0728

Artificial Intelligence Applications to Environmental Science

\section{Atmospheric Chemistry}

\section{Atmospheric Electricity}

Chairman: Dr. William R. Moninger, NOAA, R/E/FS, 325 Broadway, Boulder, CO 80303

Dr. Vernon E. Derr, NOAA, R/E, 325 Broadway, Boulder, CO 80303

Ms. Rosemary Dyer, Air Force Geophysics Laboratory, LYP, Hanscom AFB, MA 01731

Mr. J. Carr McLeod, Environment Canada, 4905 Dufferin St., Downsview, Ontario, Canada $\mathrm{M} 3 \mathrm{H} 5 \mathrm{~T} 4$

Mr. Mark Stunder, Geomet Technologies, Inc., 20251 Century Blvd., Germantown, MD 20874

Dr. Eugene S. Takle, lowa State University, 310 Curtiss Hall, Ames, IA 50011

Mr. Steven M. Zubrick, National Weather Service, NOAA, W/OM23X5, 8060 13th St., Silver Spring, MD 20910

Chairman: Prof. John T. Merrill, Graduate School of Oceanography, University of Rhode Island, South Ferry Rd., Narragansett, RI 02882-1197

Dr. Leonard A. Barrie, Atmospheric Environment Service, 4905 Dufferin St., Downsview, Ontario, Canada M3H 5T4

Dr. James E. Dye, National Center for Atmospheric Research/MMM, P.O. Box 3000, Boulder, CO 80307-3000

Dr. Donald F. Gatz, Illinois State Water Survey, 2204 Griffith Dr., Champaign, IL 61820

Dr. Dean A. Hegg, Dept. of Atmospheric Sciences, AK-40, University of Washington, Seattle, WA 98915

Dr. Joseph P. Pinto, U.S. Environmental Protection Agency, MD-80, Atmospheric Research and Exposure Assessment Laboratory, Research Triangle Park, NC 27711

Dr. Stephen E. Schwartz, Dept. of Applied Science, Environmental Chemistry Div., Brookhaven National Laboratory, Box 183, Upton, NY 11973

Chairman: Prof. Earle R. Williams, Dept. of Earth, Atmospheric, and Planetary Sciences, Massachusetts Institute of Technology, Cambridge, MA 02139

Dr. Arnold A. Barnes, Jr., AFGL/LYA, Hanscom AFB, MA 01731-5000

Dr. Richard J. Blakeslee, Mail Code ED43, Marshall Space Flight Center, NASA, Huntsville, AL 35812

Dr. John H. Helsdon, Jr., Institute of Atmospheric Sciences, South Dakota School of Mines and Technology, 501 E. St. Joseph Street, Rapid City, SD 57701-3995

Prof. Paul R. Krehbiel, Dept. of Physics, New Mexico Institute of Mining and Technology, Socorro, NM 87801

Prof. Thomas C. Marshall, Dept. of Physics and Astronomy, University of Mississippi, University, MS 38677

Dr. Vladislav Mazur, National Severe Storms Laboratory, NOAA, 1313 Halley Circle, Norman, OK 73069

Dr. Ewen Thomson, Dept. of Electrical Engineering, University of Florida, 216 Larsen Hall, Gainesville, FL 32611

Dr. John C. Willett, USAF Geophysics Laboratory, AFGL/LYA, Hanscom AFB, MA 01731

\section{Atmospheric and Oceanic Waves and Stability}

Chairman: Dr. Mankin Mak, Dept. of Atmospheric Sciences, University of Illinois at Urbana, 105 South Gregory Ave., Urbana, IL 61801

Prof. Christopher Bretherton, Dept. of Applied Meteorology, FS-20, University of Washington, Seattle, WA 98195 
Prof. Brian F. Farrell, Dept. of Earth and Planetary Sciences, Harvard University, Pierce Hall, 29 Oxford Street, Cambridge, MA 02138

Dr. Robert L. Gall, Institute of Atmospheric Physics, PAS Building \#81, Room 542, University of Arizona, Tucson, AZ 85721

Prof. Michael Ghil, Dept. of Atmospheric Sciences, University of California-Los Angeles, 405 Hilgard Ave., Los Angeles, CA 90024

Prof. W. R. Peltier, Dept. of Physics, University of Toronto, Toronto, Ontario, Canada M5S $1 \mathrm{~A} 7$

Prof. Theodore G. Shepherd, Dept. of Physics, University of Toronto, Toronto, Ontario, Canada M5S $1 \mathrm{~A} 7$

Dr. David Straus, Code 611, Laboratory for Atmospheres, Goddard Space Flight Center, NASA, Greenbelt, MD 20771

Dr. Geoffrey Vallis, Scripps Institution of Oceanography, A-021, University of California-San Diego, La Jolla, CA 92093-0209

Dr. William R. Young, Mail Code A-030, Scripps Institution of Oceanography, University of California-San Diego, La Jolla, CA 92093-0209

\section{Atmospheric Radiation}

Chairman: Prof. Graeme L. Stephens, Dept. of Atmospheric Science, Colorado State University, Fort Collins, CO 80523

Dr. Steven A. Ackerman, Cooperative Institute for Meteorological Satellite Studies, Space Science and Engineering Center, University of Wisconsin-Madison, 1225 West Dayton Street, Madison, WI 53706

Prof. Roger Davies, Dept. of Meteorology, McGill University, 805 Sherbrooke St. West, Montreal, Canada H3A 2K6

Dr. John J. DeLuisi, Geophysical Monitoring for Climatic Change, Air Research Laboratory, NOAA/ERL, R/E/CGI, 325 Broadway, Boulder, CO 80303-3328

Edwin F. Harrison, Radiation Sciences Branch, Langley Research Center, Mail Stop 420, NASA, Hampton, VA 23665-5225

Dr. Harshvardhan, Dept. of Earth and Atmospheric Sciences, Civil Engineering Bldg., Purdue University, West Lafayette, IN 47907

Dr. William B. Rossow, Goddard Institute for Space Studies, NASA, 2880 Broadway, New York, NY 10025

Dr. Francisco P. J. Valero, Atmospheric Physics Research Office, NASA/Ames Research Center, SGP: 245-4, Moffett Field, CA 94305

Dr. Warren J. Wiscombe, Code 613, Goddard Space Flight Center, NASA, Greenbelt, MD 20771

Chairman: Richard A. Wagoner, National Weather Service, NOAA, R/E/FS, 325 Broadway, Boulder, CO 80303-3328

Capt. Patrick Clyne, 34 W. Minnehaha Park, Minneapolis, MN 55419

Todd Glickman, Manager, Government Program Office, WSI Corporation, 4 Federal Street, Billerica, MA 01821

R. Craig Goff, Federal Aviation Administration Headquarters, APS 550, 800 Independence Ave., SW, Washington, DC 20591

Mr. Richard Heuwinkel, Manager, Weather Program, AOP-4, Federal Aviation Administration, Rm. 932, 800 Independence Ave., SW, Washington, DC 20591

Judson Ladd, National Weather Service Forecast Office, NOAA, 830 NE Loop 410, Suite 300, San Antonio, TX 78209

Col. John T. Madura, Commander, Det. 11, 2nd Weather Sq., Patrick AFB, FL 32925

Dr. John A. McGinley, Environmental Research Lab, NOAA, R/E/FS2, 325 South Broadway, Boulder, CO 80303-3328 
Capt. Timothy H. Miner, Executive Director, The METAR Committee, 405 Canner St., \#3, New Haven, CT 06511

James A. Moore, Terminal NEXRAD Proj. Manager, Research Applications Program, National Center for Atmospheric Research, P.O. Box 3000, Boulder, CO 80307-3000

Prof. Michael R. Poellot, Dept. of Atmospheric Sciences, University of North Dakota, Box 8216, University Station, Grand Forks, ND 58201-8216

Dr. James P. Travers, Chief, Operations Division, Office of Meteorology, National Weather Service, NOAA, Silver Spring, MD 20910

Biometeorology and Aerobiology

\section{Boundary Layers and Turbulence}

Chairman: Dr. Donald E. Aylor, Dept. of Plant Pathology and Ecology, The Connecticut Agricultural Experiment Station, P.O. Box 1106, New Haven, CT 06504

Dr. Dennis D. Baldocchi, U.S. Dept. of Commerce, National Oceanic and Atmospheric Administration, Environmental Research Laboratories, Atmospheric Turbulence and Diffusion Division, 456 South Illinois Avenue, P.O. Box 2456, Oak Ridge, TN 37831-2456

Prof. Stella Coakley, Dept. of Botany and Plant Pathology, Oregon State University, Corvallis, OR 97331

Prof. Dennis Driscoll, Dept. of Meteorology, Texas A\&M University, College Station, TX 77843-3146

Dr. Leroy Hahn, USDA Meat Animal Research Center, P.O. Box 166, Clay Center, NE 68933

Dr. Alexander MacDonald, Program for Regional Observing \& Forecasting Services, NOAA/ OAR/ERL, 325 Broadway, Boulder, CO 80303

Cynthia Rosenzweig, Goddard Institute for Space Studies, 2880 Broadway, New York, NY 10025

Dr. Peter H. Schuepp, Dept. of Renewable Resources, MacDonald College of McGill University, Ste. Anne-De-Bellevue, Quebec, Canada H9X 1C0

Dr. Roger H. Shaw, Dept. of Land, Air \& Water Resources, University of California-Davis, Davis, CA 95616

Dr. John K. Westbrook, Agricultural Research Service, USDA, Insect Biology \& Population, Management Research Lab, P.O. Box 748, Tifton, GA 31793-0748

Chairman: Prof. Christopher W. Fairall, 2740 14th Street, Boulder, CO 80302

Dr. Robert Addis, Environmental Transport Group, Environmental Technology Div., Savannah River Laboratory, E. I. duPont de Nemours \& Co., Aiken, SC 29808

Dr. Edgar L. Andreas, U.S. Army Cold Regions Research and Engineering Laboratory, 72 Lyme Road, Hanover, NH 03755-1290

Dr. Jackson Herring, National Center for Atmospheric Research, P.O. Box 3000, Boulder, CO 80307-3000

Dr. Soren E. Larsen, Dept. of Meteorology and Wind Energy, P.O. Box 49, RISO, National Laboratory, DK-4000, Roskilde, Denmark

Dr. Chin-Hoh Moeng, Mesoscale and Microscale Meteorology Division, National Center for Atmospheric Research, P.O. Box 3000, Boulder, CO 80307-3000

Dr. Carmen J. Nappo, Atmospheric Turbulence and Diffusion Division, Environmental Research Lab, NOAA, 456 South Illinois Ave., P.O. Box 2456, Oak Ridge, TN 37831-2456

Dr. James J. Riley, Mechanical Engineering Dept., FU-10, University of Washington, Seattle, WA 98195

Prof. Paul Ruscher, Dept. of Meteorology B-161, Florida State University, Tallahassee, FL 32306-3034

Dr. Ulrich Schumann, DLR, Institute of Atmospheric Physics, D-8031, Oberpfaffenhofen, Federal Republic of Germany

Chairman: Prof. Eric J. Barron, Earth Systems Science Center, 512 Deike Bldg., The Pennsylvania State University, University Park, PA 16802 
Dr. Thomas P. Ackerman, Dept. of Meteorology, Pennsylvania State University, 503 Walker Building, University Park, PA 16802

Prof. Peter Lamb, Climatology and Meteorology Section, Illinois State Water Survey, 2204 Griffith Dr., Champaign, IL 61820-7495

Dr. Ngar-Cheung Lau, Geophysical Fluid Dynamics Laboratory, P.O. Box 308, Princeton University, Princeton, NJ 08542

Dr. William Lau, Code 613, Climate and Radiation Branch, Goddard Laboratory for Atmospheres, NASA, Greenbelt, MD 20771

Prof. Gerald R. North, Dept. of Meteorology, Texas A\&M University, College Station, TX 77843-3146

Prof. V. Ramanathan, The University of Chicago, Dept. of the Geophysical Sciences, 5734 South Ellis Ave., Chicago, IL 60637

Dr. Starley Thompson, National Center for Atmospheric Research, P.O. Box 3000, Boulder, CO 80307-3000

Dr. Stephen E. Zebiak, Lamont-Doherty Geological Observatory, Columbia University, Palisades, NY 10964

\section{Cloud Physics}

\section{Hydrology}

Chairman: Prof. David Randall, Dept. of Atmospheric Science, Colorado State University, Fort Collins, CO 80523

Prof. Marcia B. Baker, Graduate Program in Geophysics/AK-50, University of Washington, Seattle, WA 98195

Prof. Kenneth V. Beard, Dept. of Atmospheric Sciences, 105 S. Gregory Ave., University of Illinois at Urbana-Champaign, Urbana, IL 61801

Prof. Judith A. Curry, Dept. of Earth and Atmospheric Sciences, Purdue University, West Lafayette, IN 47907

Dr. Andrew G. Detwiler, Institute of Atmospheric Sciences, South Dakota School of Mines and Technology, 501 E. St. Joseph Street, Rapid City, SD 57701-3995

Dr. Andrew Heymsfield, Convective Storms Division, National Center for Atmospheric Research, P.O. Box 3000, Boulder, CO 80307-3000

Dr. David O'C Starr, Code 613, Goddard Space Flight Center, NASA, Greenbelt, MD 20771

Prof. Graeme L. Stephens, Dept. of Atmospheric Sciences, Colorado State University, Fort Collins, CO 80523

Prof. Pao K. Wang, Dept. of Meteorology, University of Wisconsin-Madison, 1225 West Dayton Street, Madison, WI 53706

Chairman: Dr. Robert J. Gurney, Director, NERC Unit for Thematic Information Systems, Dept. of Geography, University of Reading, Whiteknights, P.O. Box 227, Reading, RG6 2AB, England

Prof. Rafael L. Bras, Ralph M. Parsons Laboratory, Bldg. 48-311, Massachusetts Institute of Technology, Dept. of Civil Engineering, 77 Massachusetts Ave., Cambridge, MA 02139

Dr. W.H. Brutsaert, School of Civil and Environmental Engineering, Cornell University, Hollister Hall, Ithaca, NY 14853-3501

Prof. Efi Foufoula-Georgiou, Dept. of Civil and Mineral Engineering, Mississippi River at 3rd Avenue, S.E., University of Minnesota, Minneapolis, MN 55414-2196

Dr. K. P. Georgakakos, Institute of Hydaulic Research, University of lowa, lowa City, IA 52242

Dr. William P. Kustas, USDA-ARS Hydrology Laboratory, Building 265 BARC-East, Beltsville, MD 20705

Dr. P. C. D. Milly, U.S. Geological Survey, Geophysical Fluid Dynamics Laboratory, P.O. Box 308, Princeton, NJ 08542

Dr. James A. Smith, National Weather Service, NOAA, Office of Hydrology, Rm. 530, 8060 13th St., Silver Spring, MD 20910

Prof. Soroosh Sorooshian, Dept. of Hydrology and Water Resources, College of Engineering and Mines, Bldg. II, University of Arizona, Tucson, AZ 85721 
Interaction of the

Sea and Atmosphere
Chairman: Prof. Edward S. Sarachik, Dept. of Atmospheric Sciences, AK-40, University of Washington, Seattle, WA 98195

Prof. David S. Battisti, Dept. of Meteorology, 1225 W. Dayton Street, University of WisconsinMadison, Madison, WI 53706

Dr. Mark A. Cane, Lamont-Doherty Geological Observatory of Columbia University, Route 9W, Palisades, NY 10964

Prof. Gabriel T. Csanady, Dept. of Oceanography, Old Dominion University, Norfolk, VA 23529-0276

Dr. David B. Enfield, Atlantic Oceanographic and Meteorological Lab, NOAA, 4301 Rickenbacker Causeway, Miami, FL 33149

Prof. William Large, Dept. of Oceanography, University of British Columbia, Vancouver, BC, Canada V6T 1W5

Dr. James Price, Woods Hole Oceanographic Institution, Woods Hole, MA 02543

Dr. Paul S. Schopf, Code 671, Goddard Space Flight Center, NASA, Greenbelt, MD 20771

Prof. Albert Semtner, Jr., Naval Postgraduate School, Monterey, CA 93943-5000

Chairman: Dr. Raymond M. Hoff, Atmospheric Environment Service, 4905 Dufferin St., Downsview, Ontario, Canada M3H 5T4

Dr. Wayman E. Baker, National Meteorological Center, National Weather Service, NOAA, 5200 Auth Rd., Rm. 204, Washington, DC 20233

Dr. J. Bosenberg, Max-Planck-Institut fur Meteorologie, Bundesstr. 55, D-2000 Hamburg 13, West Germany

Dr. Edward V. Browell, Mail Stop 401A, Langley Research Center, NASA, Hampton, VA 236655225

Dr. Michael Hardesty, Wave Propagation Laboratory, NOAA, 325 Broadway, Boulder, CO 80303

Dr. Dennis K. Killinger, Dept. of Physics, University of South Florida, 4202 E. Fowler Avenue, Tampa, FL 33620-5700

Dr. I. Stuart McDermid, Jet Propulsion Laboratory, Table Mountain Facility, P.O. Box 367, Wrightwood, CA 92397

Dr. John A. Reagan, Dept. of Electrical Engineering, University of Arizona, Tucson, AZ 85721

Dr. James Spinhirne, Laboratory for Atmospheres, Code 617, Goddard Space Flight Center, NASA, Greenbelt, MD 20771

Chairman: Dr. C. Bruce Baker, Meteorology Div., MD-80 Environmental Protection Agency, Research Triangle Park, NC 27711

John E. Gaynor, Environmental Research Lab, NOAA, R/E/WP7, 325 Broadway, Boulder, CO 80303

Dr. Brian G. Heikes, National Center for Atmospheric Research, P.O. Box 3000, Boulder, CO 80307

Thomas J. Lockhart, Meteorological Standards Institute, 841 Seventh Lane, P.O. Box 26, Fox Island, WA 98333

Dr. Tilden P. Meyers, Atmospheric Turbulence and Diffusion Div., Air Resource Laboratory, NOAA/OAR, Box E, Oak Ridge, TN 37831

Dr. John T. Snow, Dept. of Earth \& Atmospheric Sciences, Civil Engineering Building, Purdue University, West Lafayette, IN 47907

Dr. Kenneth H. Underwood, Director, Products Group, AeroVironment, Inc., 825 Myrtle Avenue, Monrovia, CA 91016-3424

Dr. John C. Wyngaard, National Center for Atmospheric Research, Mesoscale and Microscale Meteorology Division, P.O. Box 3000, Boulder, CO 80307-3000

Robert Young, 2801 Aero-Park Dr., Traverse City, MI 49684-9171 
Mesoscale Processes

\section{Meteorological Aspects of Air Pollution}

Chairman: Prof. Dale R. Durran, Dept. of Atmospheric Sciences, AK-40, University of Washington, Seattle, WA 98195

Prof. Peter R. Bannon, Dept. of Meteorology, 503 Walker Bldg., The Pennsylvania State University, University Park, PA 16802

Dr. Franco Einaudi, Code 612, Severe Storms Branch, Goddard Space Flight Center, NASA, Greenbelt, MD 20771

Dr. Mark R. Hjelmfelt, Institute of Atmospheric Sciences, South Dakota School of Mines and Technology, 501 E. St. Joseph Street, Rapid City, SD 57701-3995

Dr. Daniel Keyser, Dept. of Atmospheric Science, ES-224, State University of New YorkAlbany, 1400 Washington Ave., Albany, NY 12222

Dr. Ying-Hwa Kuo, National Center for Atmospheric Research, P.O. Box 3000, Boulder, CO 80307

Prof. David J. Knight, Dept. of Atmospheric Sciences, ES-228, State University of New York at Albany, 1400 Washington Ave., Albany, NY 12222

Prof. Donald J. Perkey, Dept. of Physics and Atmospheric Science, Drexel University, Philadelphia, PA 19104

Dr. Richard Rotunno, Senior Scientist, Mesoscale Prediction Section, National Center for Atmospheric Research, Mesoscale and Microscale Meteorology Division, P.O. Box 3000, Boulder, CO 80307-3000

Dr. Ronald E. Stewart, Cloud Physics Research Division, Atmospheric Environment Service, 4905 Dufferin St., Downsview, Ontario, Canada M3A 5T4

Dr. Alan J. Thorpe, Dept. of Meteorology, University of Reading, 2 Earley Gate, Reading RG6 $2 \mathrm{AU}$, United Kingdom

Chairman: Dr. Walter F. Dabberdt, National Center for Atmospheric Research, P.O. Box 3000, Boulder, CO 80307-3000

Dr. Walter D. Bach, Jr., Geosciences Division, U.S. Army Research Office, P.O. Box 12211, Research Triangle Park, NC 27709-2211

Dr. Jason Ching, Atmospheric Sciences Modeling Division, MD-80, U.S. Environmental Protection Agency, NOAA/OAR, Research Triangle Park, NC 27711

Joseph Laznow, RC Environmental Services and Technologies, P.O. Box 1500, Somerville, NJ 08876

Dr. Sharon K. LeDuc, Atmospheric Sciences Modeling Division, Atmospheric Research and Exposure Assessment Lab, MD-80, Catawba Building, 3210 Chapel Hill-Nelson Hwy., U.S. Environmental Protection Agency, Research Triangle Park, NC 27711

Dr. John Leone, Atmospheric and Geophysical Sciences Division, L-262, P.O. Box 808, Lawrence Livermore National Laboratory, Livermore, CA 94550

Dr. Leonard Levin, Electric Power Research Institute, 3412 Hillview Ave., P.O. Box 10412, Palo Alto, CA 94303

Mr. Daniel J. McNaughton, ENSR Consulting and Engineering, 95 Glastonbury Blvd., Glastonbury, CT 06033

Francis A. Schiermeier, Atmospheric Sciences Modeling Division (MD-80), Atmospheric Research and Exposure Assessment Lab., U.S. Environmental Protection Agency, Research Triangle Park, NC 27711

Dr. Jeffrey C. Weil, CIRES, Campus Box 216, University of Colorado, Boulder, CO 80309

Chairman: Prof. Steven A. Lyons, Dept. of Meteorology, College of Geosciences, Texas A\&M University, College Station, TX 77843-3146

Prof. John M. Bane, Jr., Marine Sciences Program, CB \#3300 Venable Hall, University of North Carolina-Chapel Hill, Chapel Hill, NC 27599-3300

Dr. Nancy A. Bray, Scripps Institute of Oceanography, CCS/SIO/UCSD, A-009, La Jolla, CA 92093-0209 
Lee W. Eddington, Geophysics Division, Pacific Missile Test Center, Point Mugu, CA 930425000

Dr. James E. Overland, Marine Services Research Div., NOAA/OAR, 7600 Sand Point Way NE, Seattle, WA 98115-0070

Prof. Steven R. Ramp, Dept. of Oceanography, Code 68Ra, Naval Postgraduate School, Monterey, CA 93943

Dr. David P. Rogers, Scripps Institute of Oceanography, A-021, La Jolla, CA 92093

Dr. William J. Shaw, Dept. of Meteorology, Code 63Sr, Naval Postgraduate School, Monterey, CA 93943-5000

\section{Meteorology and Oceanography of the Southern Hemisphere}

Chairman: Dr. David J. Karoly, Dept. of Mathematics, Monash University, Clayton, 3168 Victoria, Australia

Dr. Guillermo J. Berri, Departamento de Meteorologia, University of Buenos Aires, Pabellon 2, Ciudad Universitaria, 1428 Buenos Aires, Argentina

Dr. Neil Gordon, New Zealand Meteorological Service, P.O. Box 722, Wellington 1, New Zealand

Prof. Stefan L. Hastenrath, Dept. of Meteorology, University of Wisconsin-Madison, 1225 W. Dayton St., Madison, WI 53706

Dr. Vernon E. Kousky, Diagnostic Branch, W/NMC 52, Climate Analysis Center, World Weather Building, Room 605, 5200 Auth Road, Washington, DC 20233

Dr. Antonio Divino Moura, Meteorology, INPE-C. Postal 515, 12201-Sao Jose dos CamposSP, Brazil

Dr. William Randel, National Center for Atmospheric Research, P.O. Box 3000, Boulder, CO 80307-3000

Dr. Jose Rutllant, Dept. of Geophysics, University of Chile, Chile, Casilla 2777, Santiago, Chile

Prof. John Stanford, Dept. of Physics, lowa State University, Central Campus, Ames, IA 50011

Dr. Ines Velasco, Departmento de Meteorologia, Facultad de Cienceas Exactas y Naturales, Cuidad Universitaria, 1428 Buenos Aires, Argentina

Chairman: Dr. Rolando Garcia, National Center for Atmospheric Research, P.O. Box 3000, Boulder, CO 80307-3000

Prof. Susan K. Avery, Dept. of Electrical and Computer Engineering, University of Colorado, Campus Box 425, Boulder, CO 80309-2291

Dr. Guy P. Brasseur, Atmospheric Chemistry Division, National Center for Atmospheric Research, P.O. Box 3000, Boulder, CO 80307-3000

Dr. Tim Dunkerton, Northwest Research Associates, P.O. Box 3027, Bellevue, WA 98009

Dr. Kevin Hamilton, Geophysical Fluid Dynamics Laboratory, NOAA, Princeton University, P.O. Box 308, Princeton, NJ 08542

Prof. Matthew H. Hitchman, Dept. of Meteorology, University of Wisconsin-Madison, 1225 West Dayton St., Madison, WI 53706

Dr. Jeffrey T. Kiehl, National Center for Atmospheric Research, P.O. Box 3000, Boulder, CO 80307-3000

Dr. Richard Rood, Code 616, Atmospheric Chemistry and Dynamics Branch, Goddard Space Flight Center, NASA, Greenbelt, MD 20771

Dr. Anne K. Smith, Space Physics Research Laboratory, University of Michigan, Ann Arbor, MI 48109-2143

Chairman: Dr. Robert Banta, Environmental Research Lab, NOAA, R/E/WP2, 325 Broadway, Boulder, CO 80303-3328 
Prof. Peter Bannon, Dept. of Meteorology, 503 Walker Bldg., The Pennsylvania State University, University Park, PA 16802

Prof. William Blumen, Dept. of Astrogeophysics, University of Colorado, Box 391, Boulder, CO 80309

Dr. Randy Borys, Atmospheric Sciences Center, Desert Research Institute, P.O. Box 60220, Reno, NV 89506-0220

Mr. David W. Goens, Fire Weather Program, DOC/NOAA/NWS, 5225 Highway 10 West, Box 12, Missoula, MT 59802

Prof. Thomas B. McKee, Dept. of Atmospheric Science, Colorado State University, Fort Collins, CO 80523

Dr. Richard T. McNider, Research Institute-Box 212, University of Alabama in Huntsville, Huntsville, AL 35899

Dr. William M. Porch, Los Alamos National Laboratory, D466, Los Alamos, NM 87545

Dr. Piotr K. Smolarkiewicz, National Center for Atmospheric Research, Mesoscale and Microscale Meteorology Division, P.O. Box 3000, Boulder, CO 80307-3000

Dr. David Whiteman, Battelle Pacific Northwest Laboratories, Atmospheric Sciences, P.O. Box 999, Richland, WA 99352

\section{Planned and Inadvertent Weather Modification}

Chairman: Dr. David Reynolds, California Dept. of Water Resources, Rm. 606, 1416 9th St., P.O. Box 942836, Sacramento, CA 94236-0001

Dr. Bruce A. Boe, North Dakota State Water Commission, P.O. Box 1833, Bismarck, ND 58502

Dr. Larry Davis, Colorado International Corporation, P.O. Box 3007, 2851 Valmont St., Boulder, CO 80301

Dr. Arlen W. Huggins, Desert Research Institute, P.O. Box 60220, Reno, NV 89506

Dr. David B. Johnson, Applied Research Corp., 3005 Centre Green Drive, Boulder, CO 80301

Dr. L. Randall Koenig, 258 Notteargenta Road, Pacific Palisades, CA 90272

Prof. Robert M. Rauber, Dept. of Atmospheric Sciences, University of Illinois, 105 South Gregory Ave., Urbana, IL 61801

Dr. Robert F. Riggio, WHO-TV, 1801 Grand Avenue, Des Moines, IA 50308

Prof. Jeffrey L. Stith, Center for Aerospace Sciences, University of North Dakota, Box 8216 University Station, Grand Forks, ND 58202

Dr. William L. Woodley, Woodley Weather Consultants, 427 Pine Street, Boulder, CO 80302

\section{Polar Meteorology and Oceanography}

Chairman: Prof. Richard E. Moritz, Dept. of Meteorology, University of Wisconsin-Madison, 1225 West Dayton St., Madison, WI 53706

Dr. Josefino C. Comiso, Oceans and Ice Branch, Laboratory for Oceans, Code 671, Goddard Space Flight Center, NASA, Greenbelt, MD 20771

Dean Dagmar Cronn, College of Sciences, 263 Aubert Hall, University of Maine, Orono, ME 04469

Dr. Donald E. Hinsman, Sr., Scientific Officer for Satellite Activities, World Meteorological Organization, Case Postale No. 2300, CH-1211, Geneva 2, Switzerland

Prof. Lawrence A. Mysak, Dept. of Meteorology, McGill University, 805 Sherbrooke St. West, Montreal, Quebec, Canada H3A 2K6

Prof. Thomas R. Parish, Dept. of Atmospheric Science, University of Wyoming, P.O. Box 3038, University Station, Laramie, WY 82071

Dr. Carol H. Pease, Pacific Marine Environmental Research Laboratory, NOAA/OAR, BIN C15700, Building \#3, 7600 Sand Point Way, NE, Seattle, WA 98115

Dr. Donald K. Perovich, Cold Regions Research and Engineering Laboratory, 72 Lyme Road, Hanover, NY 03755

Dr. Ruth H. Preller, NORAL, Code 322, Stennis Space Center, MS 39529-5004 
Probability and Statistics

Radar Meteorology

\section{Satellite}

Meteorology and Oceanography
Chairman: Prof. Paul W. Mielke, Jr., Dept. of Statistics, Colorado State University, Fort Collins, CO 80523

Dr. Albert R. Boehm, ST Systems Corp., 109 Massachusetts Ave., Lexington, MA 02173

Dr. Tim J. Brown, National Center for Atmospheric Research, P.O. Box 3000, Boulder, CO 80307-3000

Dr. Lev S. Gandin, National Meteorological Center, W/NMC2, World Weather Building, 5200 Auth Road, Washington, DC 20233

Dr. James P. McGuirk, Dept. of Meteorology, Texas A\&M University, College Station, TX 77843-3146

Prof. Allan H. Murphy, Dept. of Atmospheric Sciences, Oregon State University, Corvallis, OR 97331-2209

Dr. Michael B. Richman, Illinois State Water Survey, 2204 Griffith Dr., Champaign, IL 618207495

Dr. H. Jean Thiebaux, National Meteorological Center, National Weather Service, NOAA, Rm. 204, 5200 Auth Rd., Washington, DC 20233

Dr. G. A. White, III, Dept. of Meteorology, Texas A\&M University, College Station, TX 77843

Dr. Raymond K. W. Wong, Resource Technologies Dept., Alberta Research Council, P.O. Box 8330, Postal Station F, Edmonton, Alberta, Canada, T6H 5X2

Chairman: Dr. Frank Marks, Hurricane Research Division, Atlantic Oceanographic and Meteorological Lab, NOAA, 4301 Rickenbacker Causeway, Miami, FL 33149

Dr. Ronald L. Alberty, Director, WSR-88D, Operational Support Facility, 1200 Westheimer Dr., Norman, OK 73069

Dr. Paul Amayenc, CNET/CRPE, Centre de Recherches en Physique de I'Environment, 38 rue du General LeClerc, 92131 Issy-les-Moulineaux, France

Dr. Edward A. Brandes, National Severe Storms Laboratory, Environmental Research Lab, NOAA, 1313 Halley Circle, Norman, OK 73069

Prof. V. N. Bringi, Dept. of Electrical Engineering, Colorado State University, Fort Collins, CO 80523

Dr. Kenneth S. Gage, NOAA/ERL, Aeronomy Laboratory, R/E/AL3, 325 Broadway, Boulder, CO 80303-3328

Dr. Jeff Keeler, Field Observing Facility, National Center for Atmospheric Research, P.O. Box 3000, Boulder, CO 80307-3000

Prof. Colleen A. Leary, Atmospheric Science Group, Box 4320, Texas Tech University, Lubbock, TX 79409

Prof. Isztar Zawadski, Departement De Physique, Universite Du Quebec a' Montreal, 1200 Alexandre, C.P. 8888, Succursale A, Montreal, Quebec, Canada H3C 3P8

Chairman: Dr. Paul Twitchell, Geophysical Sciences, 509 Schley Road, Annapolis, MD 21401

Dr. Dennis Chesters, Laboratory for Atmospheres, Code 613, Goddard Space Flight Center, NASA, Greenbelt, MD 20771

Col. Floyd Hauth, Office of Federal Coordinator for Meteorological Services and Supporting Research, Suite 300, 11426 Rockville Pike, Rockville, MD 20852

Dr. Robert E. Introne, Jr., TASC, 55 Walkers Brook Dr., Reading, MA 01867

Anthony J. Mostek, Satellite Program Leader, National Weather Service, Room 1325, 8060 13th St., Silver Spring, MD 20910

Dr. James F. W. Purdom, NOAA/NESDIS/RAMM Branch, Colorado State University, CIRA, Fort Collins, CO 80523

Dr. Larry Stowe, Atmospheric Sciences Branch, National Environmental Satellite and Data Information Service, NOAA, Rm. 711, 5200 Auth Rd., Camp Springs, MD 20746 
Dr. Gerard Szejwach, Technical Division, EUMETSAT, Am Elfengrund 45, 6100 Darmstadt, Federal Republic of Germany

\section{Severe Local Storms}

\section{Tropical Meteorology and Tropical Cyclones}

Chairman: Prof. Roger M. Wakimoto, Dept. of Atmospheric Sciences, University of California-Los Angeles, 405 Hilgard Avenue, Los Angeles, CA 90024-1565

Robert H. Johns, National Severe Storms Forecast Center, National Weather Service, NOAA, Rm. 1728, Federal Bldg., 601 E 12th St., Kansas City, MO 64106

Dr. Kevin R. Knupp, Atmospheric Science and Remote Sensing Laboratory, The University of Alabama in Huntsville, Kenneth E. Johnson Research Center, Box 212, Huntsville, AL 35899

Dr. Robert A. Maddox, National Severe Storms Laboratory, Environmental Research Lab, NOAA, 1313 Halley Circle, Norman, OK 73069

Dr. Fred Ostby, National Severe Storms Forecast Center, National Weather Service, NOAA, 601 E. 12th St., Rm. 1728, Kansas City, MO 64106

Dr. David Parsons, Mesoscale Prediction Section, National Center for Atmospheric Research, P.O. Box 3000, Boulder, CO 80307-3000

Ronald M. Reap, Office of Systems Development, National Weather Service, NOAA, Rm. 827, 8060 13th St., Silver Spring, MD 20910

Edward Szoke, National Center for Atmospheric Research/MMM, Research Lab. \#6, P.O. Box 3000, 3215 Marine St., Boulder, CO 80307-3000

Morris L. Weisman, National Center for Atmospheric Research, P.O. Box 3000, Boulder, CO 80307-3000

Ms. Marilyn M. Wolfson, (HW-25) Lincoln Laboratories, Massachusetts Institute of Technology, P.O. Box 73, Lexington, MA 02173-0073

Chairman: Dr. Stephen J. Lord, National Meteorological Center, 5200 Auth Rd., Rm. 204, National Weather Service, NOAA, Washington, DC 20233

Lt. Col. Charles P. Guard, U.S. Air Force, Joint Typhoon Warning Center, DET-\#1, 1 WW, Box 17, FPO, San Francisco, CA 96630

Prof. Richard H. Johnson, Dept. of Atmospheric Science, Colorado State University, Fort Collins, CO 80523

Prof. John Molinari, Dept. of Atmospheric Science, State University of New York at Albany, Albany, NY 12222

Dr. Lloyd J. Shapiro, Hurricane Research Div., Atlantic Oceanographic and Meteorological Lab, NOAA, 4301 Rickenbacker Causeway, Miami, FL 33149

Dr. Robert Sheets, National Hurricane Center, National Weather Service, NOAA, Rm. 631, Gables One Tower, 1320 S. Dixie Highway, Coral Gables, FL 33146-2976

Dr. Ted Z. Tsui, Environmental Prediction Research Facility, Naval Postgraduate School, Monterey, CA 93943-5009

Dr. Christopher S. Velden, Cooperative Institute for Meteorological Satellite Studies, Space Science and Engineering Center, University of Wisconsin-Madison, 1225 West Dayton Street, Madison, WI 53706

Dr. Klaus Weickmann, NOAA, Environmental Research Laboratory, 325 Broadway, Boulder, CO 80303-3328

Frank Wells, U.S. Naval Oceanographic Command Center, Joint Typhoon Warning Center, COMNAVMARIANAS, Box 17, F.P.O San Francisco, CA 96630

Chairman: Laurence G. Lee, National Weather Service Forecast Office, Raleigh-Durham International Airport, P.0. Box 80337, Raleigh, NC 27623-0337

Joseph R. Bocchieri, Office of Meteorology, National Weather Service, NOAA, 8060 13th St., Silver Spring, MD 20910

Prof. Frederick H. Carr, School of Meteorology, Rm. 219, University of Oklahoma, 200 Felgar St., Norman, OK 73019 
Gary M. Carter, National Weather Service Eastern Region (W/ER3), Airport Corporate Center, 630 Johnson Ave., Bohemia, NY 11716

Ms. Theresa R. Drake, National Weather Service Forecast Office, 192 Shafer Road, Coraopolis, PA 15108

Lawrence Dunn, National Weather Service Western Region, Scientific Services Division, P.O. Box 11188, 125 South State Street, Salt Lake City, UT 84147

Lt. Col. John L. Hayes, Dept. of the Air Force, Detachment 30, 2nd Weather Squadron, Vandenberg AFB, CA 93437-5000

Dr. L. Ray Hoxit, Certified Consulting Meteorologist, P.O. Box 836, Arden, NC 28704

Prof. Steven L. Mullen, Atmospheric Oceanic Science, University of Michigan, 2455 Hayward, Ann Arbor, Ml 48109-2143

Dr. Ralph A. Petersen, Chief, Short Range Modeling Branch, Development Division, National Meteorological Center, W/NMC22, WWB, Rm. 204, 5200 Auth Road, Room 204, Washington, DC 20233

Ronald J. Sznaider, Kavouras, Inc., Federal Aviation Bldg., 6301 34th Ave. South, Minneapolis, MN 55450

Jeffrey P. Walker, National Weather Service Western Region, NOAA, Box 11188, Federal Bldg., 125 S. State St., Salt Lake City, UT 84147

\section{EDUCATION AND MANPOWER COMMISSION}

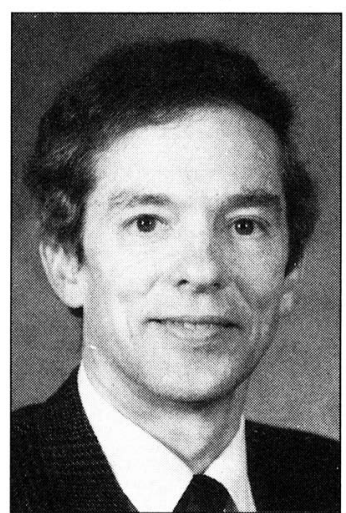

David D. Houghton
Chairman: Prof. David D. Houghton, Dept. of Meteorology, University of Wisconsin-Madison, 1225 West Dayton St., Madison, WI 53706

Members: Chairmen of the constituent Boards

\section{Board of Meteorological and Oceanographic Education in Universities}

Chairman: Prof. Dayton Vincent, Dept. of Atmospheric Sciences, Purdue University, West Lafayette, IN 47907

Prof. Howard Bluestein, School of Meteorology, University of Oklahoma, Norman, OK 73019

Robert Bunting, University Corporation for Atmospheric Research, P.O. Box 3000, Boulder, CO 80307

Terrance Clark, 3350th TCHTG/TTMV, Chanute AFB, IL 61868

Dr. Joseph Klemp, National Center for Atmospheric Research, P.O. Box 3000, Boulder, CO 80307

Dr. John McCarthy, National Center for Atmospheric Research, P.O. Box 3000, Boulder, CO 80307

Dr. Charles H. Peterson, Institute of Marine Science, University of North Carolina, Chapel Hill, Morehead City, NC 28557-0809

Dr. Timothy Spangler, COMET, University Corporation for Atmospheric Research, P.O. Box 3000, Boulder, CO 80307-3000 
David M. Straus, COLA, Dept. of Meteorology, University of Maryland, College Park, MD 20742

Richard Wagoner, National Weather Service, NOAA, R/E/FS, 325 Broadway, Boulder, CO 80303-3328

Prof. John W. Winchester, Dept. of Oceanography, Florida State University, Tallahassee, FL 32306

Prof. Joe Zabransky, Natural Science Dept., Plymouth State College, College Park, NH 03264

\section{Board of School and Popular Meteorological and Oceanographic Education}

Chairman: Prof. John T. Snow, Dept. of Earth and Atmospheric Sciences, Purdue University, West Lafayette, IN 47907

Robert Breck, WVUE-TV, 1025 S. Jefferson Davis Parkway, New Orleans, LA 70125

Peter Giddings, ABC/CCCI KGO-TV, 900 Front St., San Francisco, CA 94111-1450

Ernest Kern, Earth Science Teacher Training Center, Southeast Missouri State University, Cape Girardeau, MO 63701

Mike Passow, White Plains Middle School, 7 Amherst Place, White Plains, NY 10601

Steven Richards, NE Bronx Education Park, NE Bronx Weather Station, 750 Baychester Ave., Room 228, Bronx, NY 10475

Bruce Schwoegler, WBZ-TV 4, 1170 Soldier's Field Rd., Boston, MA 02134

Prof. David R. Smith, Dept. of Oceanography, U.S. Naval Academy, Annapolis, MD 21402

Steven D. Steinke, Association of American Weather Observers, P.O. Box 455, Belvidere, IL 61008

Prof. Naomi Surgi, RSMAS/MPO, University of Miami, 4600 Rickenbacker Causeway, Miami, FL 33149

Brian Sussman, KPIX-TV, 855 Battery, San Francisco, CA 94111

Prof. Linda Whittaker Keller, Dept. of Meteorology, University of Wisconsin-Madison, 1225 W. Dayton St., Madison, WI 53706

Donald Witten, Public Affairs Officer/NWS, 620 Gramax Bldg., Silver Spring, MD 20910

Chairman: Prof. Russell DeSouza, Dept. of Earth Sciences, Millersville University of Pennsylvania, Millersville, PA 17551

Prof. Frank Colby, Dept. of Earth Sciences, University of Lowell, Lowell, MA 01854

Prof. Phil Smith, Dept. of Earth and Atmospheric Sciences, Purdue University, West Lafayette, IN 47907

Chairman: Edward Young, Jr., Pacific Region Headquarters, National Weather Service, NOAA, 300 Ala Moana Blvd., Rm. 4110, Honolulu, HI 96850-0002

Bill Beams, National Weather Service Office, P.O. Box 321, Yakutat, AK 99689

Valerie Voss Collins, CNN-News, One CNN Ctr., P.O. Box 105366, Atlanta, GA 30348-5366

Al Druemont, National Weather Service Forecast Office, North Crown Bldg., 830, NE Loop 410, Suite 300, San Antonio, TX 78209

Renee R. Fair, National Weather Service Forecast Office, NOAA, Massachusetts Technology Center, Suite 102N, Logan International Airport, 1 Harborside Dr., East Boston, MA 02128

Dr. Rex Fleming, 4889 Country Club Way, Boulder, CO 80301

Eugenia Kalnay, National Meteorological Center, National Weather Service, NOAA, W/NMC22, World Weather Bldg., Rm. 204, Washington, DC 20233

Prof. Lonzy Lewis, Dept. of Physics and Atmospheric Sciences, Jackson State University, Jackson, MI 39217

Prof. Kenneth P. MacKay, Professor of Meteorology, San Jose State University, One Washington Square, San Jose, CA 95192-0104 
Dr. Kingtse Mo, Climate Analysis Center, National Weather Service, NOAA, W/NMC52, World Weather Bldg., Rm. 606, Washington, DC 20233

Kaye O'Brien, EEO Coordinator, Western Region Headquarters, National Weather Service, NOAA, P.O. Box 11188, Federal Building, Salt Lake City, UT 84147

Prof. Patricia Pauley, Dept. of Meteorology, University of Wisconsin, 1225 West Dayton St., Madison, WI 53706

Major Carol Belt Weaver, Headquarters Air Weather Service/DOSP, Scott AFB, IL 62225-5008

Dr. Susan F. Zevin, Eastern Region, National Weather Service, NOAA, Airport Corporate Ctr., 630 Johnson Ave., Bohemia, NY 11716

\section{PLANNING COMMISSION}

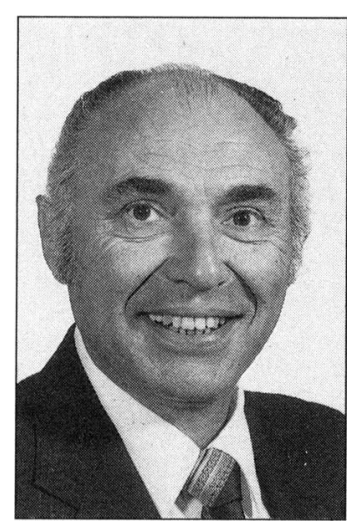

Clifford J. Murino
Chairman: Dr. Clifford J. Murino, 248 East Bain Dr., Tidewater, OR 97390

Dr. Eugene W. Bierly, Director, Division of Atmospheric Sciences, National Science Foundation, 1800 G Street, NW, Rm. 644, Washington, DC 20550

Prof. Roscoe R. Braham, Jr., Dept. of Geophysical Sciences, The University of Chicago, 5734 South Ellis Ave., Chicago, IL 60637

David S. Johnson, Study Director, National Weather Service Modernization Committee, National Research Council (HA292B), 2101 Constitution Ave., Washington, DC 20418

Roy L. Leep, WTVT Television Weather Service, P.O. Box 3113, Tampa, FL 33631-3113

Prof. Richard J. Reed, Dept. of Atmospheric Sciences, University of Washington, Seattle, WA 98195

Dr. Joanne Simpson, Chief Scientist for Meteorology, Code 612, Laboratory for Atmospheres, Goddard Space Flight Center, NASA, Greenbelt, MD 20771 